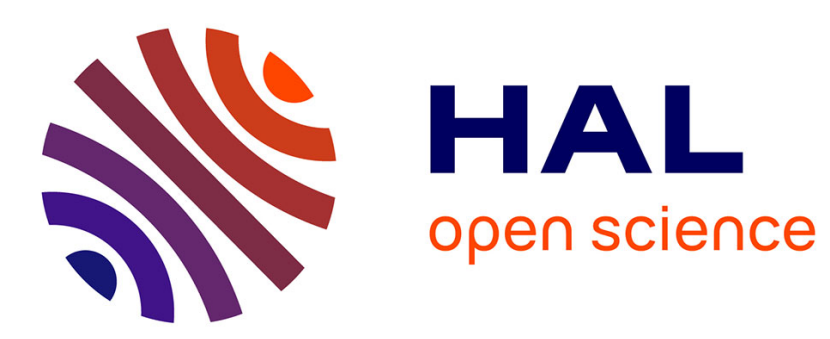

\title{
Heat kernel and Green function estimates on noncompact symmetric spaces II
}

\author{
Jean-Philippe Anker, Lizhen Ji
}

\section{To cite this version:}

Jean-Philippe Anker, Lizhen Ji. Heat kernel and Green function estimates on noncompact symmetric spaces II. 2001, pp.1-9. hal-00022965

\section{HAL Id: hal-00022965 \\ https://hal.science/hal-00022965}

Submitted on 17 Apr 2006

HAL is a multi-disciplinary open access archive for the deposit and dissemination of scientific research documents, whether they are published or not. The documents may come from teaching and research institutions in France or abroad, or from public or private research centers.
L'archive ouverte pluridisciplinaire HAL, est destinée au dépôt et à la diffusion de documents scientifiques de niveau recherche, publiés ou non, émanant des établissements d'enseignement et de recherche français ou étrangers, des laboratoires publics ou privés. 


\title{
HEAT KERNEL AND GREEN FUNCTION ESTIMATES ON NONCOMPACT SYMMETRIC SPACES II
}

\author{
JEAN-PHILIPPE ANKER \& LIZHEN Ji
}

\author{
published in \\ Topics in probability and Lie groups, J. C. Taylor (ed.), \\ CRM Proc. Lect. Notes 28, Amer. Math. Soc. (2001), 1-9
}

\section{$\S 1$. INTRODUCTION}

For a complete Riemannian manifold, the heat kernel and Green function are two natural and important spectral functions. They play an important role in studying the spectrum of the manifold and understanding relations between the spectrum and geometry (see $[\mathrm{C}],[\mathrm{D}],[\mathrm{G}],[\mathrm{BGV}]$ and the references there).

Numerous results have been obtained for the heat kernel and Green function, in particular various Gauss type bounds on the heat kernel of noncompact manifolds (see $[G]$ ). For general Riemannian manifolds, the bounds on the heat kernel are better when the manifolds are nonnegatively curved, though the lower and upper bounds are not of the same order (see [LY] for example).

Riemannian symmetric spaces of noncompact type form an important class of nonpositively curved, simply connected Riemannian manifolds. For such spaces, the heat kernel has been studied intensively using techniques from harmonic analysis on Lie groups; better bounds on the heat kernel are known, and sharp bounds have been obtained for some special spaces, for example, hyperbolic spaces (see [A2], [A3], [AJ1], [D], and the references there).

In [AJ1], for all noncompact symmetric spaces, we obtain optimal upper and lower bounds on and exact asymptotics of the heat kernel in the restricted region where the space variable is bounded by an arbitrary large multiple of the time variable, which

1991 Mathematics Subject Classification. 22E30, 22E46, 31C12, 43A80, 43A85, 43A90, 58G11.

Key words and phrases. Green function, Harnack inequality, heat kernel, semisimple Lie groups, spherical functions, symmetric spaces (Riemannian, noncompact).

First author partially supported by the European Commission (HCM Network 1994-1997 Fourier Analysis and TMR Network 1998-2001 Harmonic Analysis). Second author partially supported by the U.S.A. National Science Foundation (postdoctoral fellowship DMS 9407427 and grant DMS 9704434)

Typeset by $\mathcal{A}_{\mathcal{M}} \mathcal{S}-\mathrm{TEX}_{\mathrm{E}}$ 
is critical for many applications. For example, using these bounds on the heat kernel, we obtain sharp bounds on the Green function, which are used in an essential way in [GJT, Chap. VIII] to determine the Martin compactification of symmetric spaces. Other applications include sharp bounds on the Poisson kernel, the weak type $(1,1)$ inequality for the heat maximal operator on Iwasawa $A N$ groups, and the $L^{p}$ heat propagation (see [AJ1, §4] for details). We will also comment in $\S 2$ below on implications of our results for large time behaviors of the heat kernel as opposed to the well-known small time asymptotics of the heat kernel.

When the isometry group of the symmetric spaces is a complex Lie group, the heat kernel can be computed in terms of elementary functions, and the sharp bounds and the exact asymptotics can be derived easily. Otherwise, the Fourier transformation of the symmetric spaces expresses the heat kernel as an integral involving non-elementary spherical functions and the Harish-Chandra $\mathbf{c}-$ function, and the problem is to estimate this integral. For higher rank symmetric spaces, one of the difficulties in estimating the heat kernel lies along the walls of the Weyl chamber. For this purpose, we use the complicated asymptotic expansion of spherical functions along the walls developed by Trombi and Varadarajan [TV].

The main purpose of this note is to give an alternative approach to obtain the sharp bounds without using this asymptotic expansion. Specifically, in [AJ1], we obtained the sharp bounds on the heat kernel away from the walls using only the Harish-Chandra convergent expansion of spherical functions inside the Weyl chamber. The result in this note is that the sharp bounds away from the walls combined with the parabolic Harnack inequality of Li and Yau [LY] imply the sharp bounds on the heat kernel in the above restricted region. We also outline another approach to the asymptotics of and hence the sharp bounds on the the heat kernel away from the walls, thus making this note basically self-contained.

The basic point of this note is that the analysis of spherical functions near the walls is very subtle, but the heat kernel behaves nicely because it satisfies the parabolic Harnack inequality. On the other hand, Trombi and Varadarajan's asymptotic expansion of spherical functions along the walls is essential for the asymptotics of the heat kernel and the Green function along the walls (see $[\mathrm{AJ} 1, \S 5]$ ).

We would like to point out that the result of this note was not announced at this conference in 1992 and hence does not correspond to a talk by either of us at the meeting. In fact, this simplification of going over the walls via the Harnack inequality was observed in 1997 when [AJ1] was almost written up. We would like to thank J. C. Taylor for suggesting to include this note into this volume.

\section{$\S 2$. Sharp bounds on the heat Kernel and the Green function}

We recall several notations from $[\mathrm{AJ} 1, \S 2]$. Let $G$ be a connected semisimple Lie group of noncompact type with finite center, $K \subset G$ a maximal compact subgroup, and $\mathbb{X}=G / K$ the associated symmetric space. Let $\mathfrak{a} \subset \mathfrak{g}$ be a Cartan subspace, and $\Sigma=\Sigma(\mathfrak{g}, \mathfrak{a})$ the set of restricted roots. Fix a positive Weyl chamber $\mathfrak{a}^{+}$, and denote by $\Sigma^{+}$(resp. $\Sigma^{++}$) the set of positive (resp. positive indivisible) roots. 
Let $h_{t}\left(y^{-1} x\right)=h_{t}(x, y)$ be the heat kernel of $\mathbb{X}$. Then one of the main results in [AJ1, Theorem 3.7.ii] is the following sharp bounds.

Theorem 2.1. For any $\kappa>0$, there exist positive constants $C_{1}, C_{2}$ such that, for any $H \in \overline{\mathfrak{a}^{+}}$with $|H| \leq \kappa(1+t)$,

$$
C_{1} \leq \frac{h_{t}(\exp H)}{t^{-\frac{n}{2}}(1+t)^{\frac{m}{2}-\left|\Sigma^{++}\right|}\left\{\prod_{\alpha \in \Sigma^{++}}(1+\langle\alpha, H\rangle)\right\} e^{-|\rho|^{2} t-\langle\rho, H\rangle-\frac{|H|^{2}}{4 t}}} \leq C_{2},
$$

where $n=\operatorname{dim} \mathbb{X}, m$ is equal to the sum of dimensions of the positive root spaces $\mathfrak{g}_{\alpha}$ and $\rho$ is half sum of the positive roots with multiplicity.

Let $\Delta$ be the Laplace-Beltrami operator of $\mathbb{X}$, which is taken to be nonpositive, and $k_{\zeta}$ the kernel of $\left(-\Delta-|\rho|^{2}+\zeta^{2}\right)^{-1}$, i.e. the Green function of $\mathbb{X}$. By the relation

$$
\left(-\Delta-|\rho|^{2}+\zeta^{2}\right)^{-1}=\int_{0}^{\infty} d t e^{\left(|\rho|^{2}-\zeta^{2}\right) t} e^{t \Delta}
$$

the above sharp bounds on the heat kernel imply the following sharp bounds on the Green function $k_{\zeta}$ (see [AJ1, §4] for details).

Corollary 2.2. For any $\zeta>0$, there exist positive constants $C_{1}, C_{2}$ such that, for any $H \in \overline{\mathfrak{a}^{+}}$with $|H| \geq 1$,

$$
C_{1} \leq \frac{k_{\zeta}(\exp H)}{|H|^{\frac{1-\ell}{2}-\left|\Sigma^{++}\right|} \varphi_{0}(\exp H) e^{-\zeta|H|}} \leq C_{2},
$$

where $\ell=\operatorname{dim} \mathfrak{a}$, the rank of $\mathbb{X}$.

Using the sharp bounds on $\varphi_{0}(\exp H)$ in [A1], the sharp bounds on the Green function are equivalent to the following:

$$
C_{1} \leq \frac{k_{\zeta}(\exp H)}{|H|^{\frac{1-\ell}{2}-\left|\Sigma^{++}\right|} \prod_{\alpha \in \Sigma^{++}}(1+\langle\alpha, H\rangle) e^{-\langle\rho, H\rangle-\zeta|H|}} \leq C_{2} .
$$

The exact asymptotics of the heat kernel $h_{t}(\exp H)$ and hence the Green function $k_{\zeta}(\exp H)$ are also obtained in $[\mathrm{AJ} 1, \S 5]$. Sharp bounds and asymptotics for $k_{\zeta}$ when $\zeta=0$ are obtained in [AJ1] as well. Since only the sharp bounds on the Green function $k_{\zeta}$ for $\zeta>0$ are used in [GJT] to determine the Martin compactification of $\mathbb{X}$, we will not state them here.

We would also like to point out that the Martin compactification is defined in terms of the asymptotics at infinity of the normalized Green function (see [GJT, Chap. VI] or the survey article by Taylor [T] in this volume), and the asymptotic results in [AJ1, §5] could simplify the arguments in [GJT, Chap. VIII] in determining the Martin compactification. On the other hand, this note shows that it is much easier, at least technically, to obtain the sharp bounds on the Green function than the exact asymptotics, and hence the arguments in [GJT, Chap. VIII] are fully justified.

Another consequence of Theorem 2.1 is the following sharp bounds on the large time asymptotics of the heat kernel $h_{t}(\exp H)$. 
Corollary 2.3. For any fixed $H \in \mathfrak{a}$ and $t \geq 1$, there exists positive constants $C_{1}, C_{2}$ such that

$$
C_{1} \leq \frac{h_{t}(\exp H)}{t^{-\frac{\ell}{2}-\left|\Sigma^{++}\right|} e^{-|\rho|^{2} t-\frac{|H|^{2}}{4 t}}} \leq C_{2}
$$

As mentioned above, the exact asymptotics as $t \rightarrow+\infty$ are also available. This result sheds some lights on the large time asymptotics of the heat kernel of general Riemannian manifolds.

It is well-known that for, any Riemannian manifold, the heat kernel has a small time asymptotic expansion, and the small time behavior determines the Weyl law on distribution of eigenvalues when the manifold is compact, and the relation between the small and large time behaviors of the heat kernel plays an important role in the heat kernel proof of the Atiyah-Singer index theorem (see [BGV] for example).

Inspired by the importance of the small time asymptotics, a natural question is whether the heat kernel admits large time asymptotics. In fact, large time behaviors of the heat kernel of the universal covering space of a compact Riemannian manifold is closely related to the so-called Novikov-Shubin invariants (see $[\mathrm{L}]$ ). For certain Lie groups, large time asymptotics of the heat kernel of more general operators have been studied intensitively by Varopoulos and his school using probability methods (see [Va] and the references there).

The small time asymptotics resemble the heat kernel of the Euclidean space $\mathbb{R}^{n}$, which is given by the Gauss kernel

$$
h_{t}(x, y)=(4 \pi t)^{-\frac{n}{2}} e^{-\frac{|x-y|^{2}}{4 t}} .
$$

On the other hand, the large time behaviors could be quite different. In fact, the results in Corollary 2.3 suggest the following spectral explanation of some possible terms appearing in the large time asymptotics of the heat kernel of a Riemannian manifold: (1) When the bottom of the spectrum $\lambda_{0}$ of the negative of the Laplace operator $\Delta$ is strictly positive, there is an additional exponential decaying term $e^{-\lambda_{0} t}$. (2) The exponent of the additional polynomial decay is determined by the dimension of the continuous spectrum of $-\Delta$ near the bottom $\lambda_{0}$ and the vanishing order of the spectral measure at the bottom. For some solvable Lie groups, there could be additional sub-exponential and sup-polynomial decaying terms (see [Va]), whose spectral meaning is not clear yet.

For noncompact symmetric spaces, $\lambda_{0}=|\rho|^{2}$; and the spectrum of $-\Delta$ is purely continuous and has dimension equal to $\ell=\operatorname{dim} \mathfrak{a}$; and the spectral measure (or the Plancherel measure) is given by the negative square of Harish-Chandra's c-function and its vanishing order at the origin is equal to $2\left|\Sigma^{++}\right|$.

Now we summarize very briefly the approach in [AJ1]. Using the spherical Fourier transform on $G$, the heat kernel $h_{t}(\exp H), H \in \mathfrak{a}$, can be written as

$$
h_{t}(\exp H)=c_{2} \int_{\mathfrak{a}^{+}} \frac{d \lambda}{|\mathbf{c}(\lambda)|^{2}} e^{-t\left(|\lambda|^{2}+|\rho|^{2}\right)} \varphi_{\lambda}(\exp H)
$$


where $\varphi_{\lambda}(\exp H)$ is the spherical function, $\mathbf{c}(\lambda)$ is Harish-Chandra's $\mathbf{c}-$ function, and $c_{2}$ is the positive constant entering the inversion formula for the spherical Fourier transform in [AJ1, Theorem 2.2.2.ii].

The basic idea in the proof of [AJ1, Theorem 3.7] is to expand $\varphi_{\lambda}(\exp H)$ in terms of exponential functions and estimate $h_{t}(\exp H)$ differently depending on the position of $H$ relative to the walls of the Weyl chamber. When $H$ is close to the walls, an important technique is induction on the semisimple rank (the descent argument). It is here that Trombi and Varadarajan's asymptotic expansion plays a crucial role. Because no good bounds on the terms in the asymptotic expansion are available and this asymptotic expansion may not converge, the condition $|H| \leq \kappa(1+t)$ is imposed in the above theorem.

As mentioned earlier, the main purpose of this note is to give an alternative proof of this theorem without using Trombi and Varadarajan's asymptotic expansion of spherical functions.

\section{§3. Sharp bOUnds AND ASYMPtotics AWAY FROM THE WALLS}

In [AJ1, Theorem 3.7.i], we obtained the following sharp bounds on the heat kernel away from the walls of the Weyl chamber using only Harish-Chandra's convergent expansion of spherical functions inside the Weyl chamber.

Proposition 3.1. (Bounds away from the walls) There exist positive constants $\omega, C_{1}$, $C_{2}$ such that, for every $H \in \mathfrak{a}^{+}$with $\inf _{\alpha \in \Sigma^{+}}\langle\alpha, H\rangle \geq \omega$, and all $t \geq \omega$,

$$
C_{1} \leq \frac{h_{t}(\exp H)}{t^{-\frac{n}{2}}\left\{\prod_{\alpha \in \Sigma^{++}}\langle\alpha, H\rangle(t+\langle\alpha, H\rangle)^{\frac{m_{\alpha}+m_{2 \alpha}}{2}-1}\right\} e^{-|\rho|^{2} t-\langle\rho, H\rangle-\frac{|H|^{2}}{4 t}}} \leq C_{2}
$$

The upper bound is proved in Step 2 of the proof of Theorem 3.7 in [AJ1], and the lower bound in Step 6. Though the proof is complicated, the basic idea is simple and can be outlined as follows.

When $H \in \mathfrak{a}^{+}$and $\lambda$ is regular, we have Harish-Chandra's converging expansion of spherical function $\varphi_{\lambda}(\exp H)$ in terms of exponential functions (cf. [AJ1, Theorem 2.2.8]):

$$
\varphi_{\lambda}(\exp H)=e^{-\langle\rho, H\rangle} \sum_{q \in 2 Q} e^{-\langle q, H\rangle} \sum_{w \in W} \mathbf{c}(w \lambda) \gamma_{q}(w \lambda) e^{i\langle w \lambda, H\rangle} .
$$

Then $h_{t}(\exp H)$ is the sum of the terms

$$
c_{2} e^{-|\rho|^{2} t-\langle\rho, H\rangle-\langle q, H\rangle} \int_{\mathfrak{a}} d \lambda \mathbf{c}(-\lambda)^{-1} \gamma_{q}(\lambda) e^{-t|\lambda|^{2}+i\langle\lambda, H\rangle} .
$$

These terms can be estimated in two steps: (1) shift the contour of integration from $\mathfrak{a}$ to $\mathfrak{a}+i \frac{H}{2 t}$ to produce the expected Gaussian factor $e^{-\frac{|H|^{2}}{4 t}},(2)$ set the integration variable $\lambda=0$ in non-elementary factors in the integrand to get the leading term. 
The crucial point is to justify Step 2 and show that, for $q \in 2 Q, q \neq 0$, the term contributing to $h_{t}(\exp H)$ is of smaller order. For details, see Steps 2 and 6 of the proof of [AJ1, Theorem 3.7].

In the following, we outline an alternative proof of Proposition 3.1 and hence of Theorem 2.1.

We first obtain the asymptotics of the heat kernel $h_{t}(\exp H)$ away from the walls [AJ1, Theorem 5.1.1.i] using a different method and then derive the sharp bounds in Proposition 3.1. The proof of [AJ1, Theorem 5.1.1] depends on the proof of the sharp bounds in [AJ1, Theorem 3.7].

Proposition 3.2. (Asymptotics away from the walls) Let $H_{j} \in \mathfrak{a}^{+}$be a unbounded sequence. If $\left\langle\alpha, H_{j}\right\rangle \rightarrow+\infty$ for all $\alpha \in \Sigma^{+}$and $t_{j} \rightarrow+\infty$, then $^{1}$

$$
h_{t_{j}}\left(\exp H_{j}\right) \sim c_{2} \pi^{\frac{\ell}{2}} t_{j}^{-\frac{\ell}{2}} \mathbf{c}\left(-\frac{i H_{j}}{2 t_{j}}\right)^{-1} e^{-|\rho|^{2} t_{j}-\left\langle\rho, H_{j}\right\rangle-\frac{\left|H_{j}\right|^{2}}{4 t_{j}}},
$$

where $\ell=\operatorname{dim} \mathfrak{a}$ is the rank of $\mathbb{X}, \mathbf{c}(\cdot)$ is Harish-Chandra's function, and $c_{2}$ is the same positive constant as above.

Proof. As mentioned earlier, we have

$$
h_{t_{j}}\left(\exp H_{j}\right)=c_{2} e^{-|\rho|^{2} t_{j}-\left\langle\rho, H_{j}\right\rangle} \sum_{q \in 2 Q} e^{-\left\langle q, H_{j}\right\rangle} \int_{\mathfrak{a}} d \lambda \mathbf{c}(-\lambda)^{-1} \gamma_{q}(\lambda) e^{-|\lambda|^{2} t_{j}+i\left\langle\lambda, H_{j}\right\rangle} .
$$

Because of the decaying factor $e^{-\left\langle q, H_{j}\right\rangle}$ for $q \neq 0$, the leading term of $h_{t_{j}}\left(\exp H_{j}\right)$ can be shown to be equal to

$$
c_{2} e^{-|\rho|^{2} t_{j}-\left\langle\rho, H_{j}\right\rangle} \int_{\mathfrak{a}} d \lambda \mathbf{c}(-\lambda)^{-1} e^{-|\lambda|^{2} t_{j}} e^{i\left\langle\lambda, H_{j}\right\rangle} .
$$

If $\mathbf{c}(-\lambda)^{-1}$ is a polynomial in $\lambda$, then we can compute this term explicitly in terms of elementary functions. But, in general, $\mathbf{c}(-\lambda)^{-1}$ only has a polynomial growth. ${ }^{2}$ The basic idea is to replace $\mathbf{c}(-\lambda)^{-1}$ by a polynomial and estimate the error thus arising. More precisely, define a polynomial

$$
P(\lambda)=\prod_{\alpha \in \Sigma^{++}}-i\langle\alpha, \lambda\rangle(1-i\langle\alpha, \lambda\rangle)^{k}
$$

where $k>\frac{m_{\alpha}+m_{2 \alpha}}{2}$ for all $\alpha$. Let $f(\lambda)=\mathbf{c}(-\lambda)^{-1} / P(\lambda)$. Then $\mathbf{c}(-\lambda)^{-1}=P(\lambda) f(\lambda)$. Define the Fourier transform

$$
\hat{f}(H)=\int_{\mathfrak{a}} d \lambda f(\lambda) e^{i\langle\lambda, H\rangle} .
$$

\footnotetext{
${ }^{1}$ For two functions $f(j)$ and $g(j)$ of $j$, the expression $f(j) \sim g(j)$ means that $\lim _{j \rightarrow+\infty} f(j) / g(j)=1$.

${ }^{2}$ If $G$ is a complex Lie group, then $\mathbf{c}(\lambda)^{-1}$ is a polynomial and $|\mathbf{c}(\lambda)|^{-2} \varphi_{\lambda}(\exp H)$ is an elementary function of $\lambda$, and hence $h_{t}(\exp H)$ can be computed explicitly.
} 
Then $\hat{f}(H)$ is a bounded continuous function. Since both $\mathbf{c}(-\lambda)^{-1}$ and $1 / P(\lambda)$ are holomorphic on the tubular cone $\mathfrak{a}+i \mathfrak{a}^{+}, f(\lambda)$ is holomorphic on the tubular cone $\mathfrak{a}+i \mathfrak{a}^{+}$. Therefore $f(\lambda)$ belongs to the class $H_{0}\left(0 ; \mathfrak{a}^{+}\right)$, according to the definition of $H_{p}(a ; C)$ in [Vl, p. 238]. By Theorem 2 and Corollary 1 in [Vl, p. 239], the support of $\hat{f}(H)$ is contained in $-\overline{{ }^{+} \mathfrak{a}}$, where ${ }^{+} \mathfrak{a}=\left\{\lambda \in \mathfrak{a} \mid\langle\lambda, H\rangle>0\right.$, for all $\left.H \in \mathfrak{a}^{+}\right\}$, the so-called big chamber dual to the positive Weyl chamber $\mathfrak{a}^{+}$.

The Fourier transform of $P(\lambda) e^{-|\lambda|^{2} t}$, denoted by $F(H, t)$, is given by

$$
F(H, t)=(4 \pi t)^{-\frac{\ell}{2}} \prod_{\alpha \in \Sigma^{++}}-\partial_{\alpha}\left(1-\partial_{\alpha}\right)^{k} e^{-\frac{|H|^{2}}{4 t}} .
$$

Then

$$
\int_{\mathfrak{a}} d \lambda \mathbf{c}(-\lambda)^{-1} e^{-|\lambda|^{2} t_{j}-i\left\langle\lambda, H_{j}\right\rangle}=(2 \pi)^{\ell} \int_{-\overline{{ }_{\mathfrak{a}}}} d v F\left(H_{j}-v, t_{j}\right) \hat{f}(v) .
$$

Using the fact that $\prod_{\alpha \in \Sigma^{++}}\langle\alpha, H\rangle$ is a skew-symmetric polynomial on $\mathfrak{a}$ under the Weyl group $W$ of the least degree, we can show that as $j \rightarrow+\infty$,

$$
F\left(H_{j}-v, t_{j}\right) \sim\left(4 \pi t_{j}\right)^{-\frac{\ell}{2}} \prod_{\alpha \in \Sigma^{++}} \frac{\left\langle\alpha, H_{j}\right\rangle}{2 t_{j}}\left(1+\frac{\left\langle\alpha, H_{j}\right\rangle}{2 t_{j}}\right)^{k} e^{-\frac{\left|H_{j}\right|^{2}}{4 t_{j}}-\frac{|v|^{2}}{4 t_{j}}-\frac{\left\langle H_{j}, v\right\rangle}{2 t_{j}}},
$$

and hence

$$
F\left(H_{j}-v, t_{j}\right) \sim\left(4 \pi t_{j}\right)^{-\frac{\ell}{2}} \prod_{\alpha \in \Sigma^{++}} \frac{\left\langle\alpha, H_{j}\right\rangle}{2 t_{j}}\left(1+\frac{\left\langle\alpha, H_{j}\right\rangle}{2 t_{j}}\right)^{k} e^{-\frac{\left|H_{j}\right|^{2}}{4 t_{j}}+\frac{\left\langle H_{j}, v\right\rangle}{2 t_{j}}} .
$$

By changing the order of this limit and the integration, which can be justified, we get the desired asymptotics of $h_{t_{j}}\left(\exp H_{j}\right)$.

Proof of Proposition 3.1. From this asymptotic result in Proposition 3.2, we get the following alternative proof of Proposition 3.1. By contradiction, we can show that there exist positive constants $C_{1}, C_{2}$ and a large positive constant $\omega$ such that, for every $H \in \mathfrak{a}^{+}$with $\inf _{\alpha \in \Sigma^{+}}\langle\alpha, H\rangle \geq \omega$ and all $t \geq \omega$,

$$
C_{1} \leq \frac{h_{t}(\exp H)}{t^{-\frac{\ell}{2}} \mathbf{c}\left(-\frac{i H}{2 t}\right)^{-1} \exp \left(-|\rho|^{2} t-\langle\rho, H\rangle-\frac{|H|^{2}}{4 t}\right)} \leq C_{2} .
$$

Then the proposition follows from the following two results: for $H \in \mathfrak{a}^{+}$,

$$
\phi_{0}(\exp H) \asymp\left\{\prod_{\alpha \in \Sigma^{++}}(1+\langle\alpha, H\rangle)\right\} e^{-\langle\rho, H\rangle}
$$

(see $[\mathrm{A} 1])$, and

$$
\prod_{\alpha \in \Sigma^{++}} \frac{\langle\alpha, H\rangle}{t}\left(1+\frac{\langle\alpha, H\rangle}{t}\right)^{\frac{m_{\alpha}+m_{2 \alpha}}{2}-1} \asymp \mathbf{c}\left(-\frac{i H}{2 t}\right)^{-1}
$$

where $m_{\alpha}=\operatorname{dim} \mathfrak{g}_{\alpha} \cdot{ }^{3}$

\footnotetext{
${ }^{3}$ For two functions $f$ and $g$, the expression $f \asymp g$ means that there exist two positive constants $C_{1}$, $C_{2}$ such that $C_{1} f \leq g \leq C_{2} f$.
} 


\section{$\S 4$. The Harnack INEQUALity AND SHARP BOUNDS ALONG THE WALLS}

To extend the sharp bounds on the heat kernel in Proposition 3. to the walls of the Weyl chamber, we need the following Harnack inequality of Li and Yau [LY, Theorem 2.2], [SY, Theorem 2.2 on p. 163].

Proposition 4.1. Let $M$ be a n-dimensional complete, noncompact Riemannian manifold with Ricci curvature Ric $(M) \geq-k$, for some $k \geq 0$. If $u(x, t)$ is a positive solution of the heat equation on $M$, then, for any $a>1, x_{1}, x_{2} \in M, 0<t_{1}<t_{2}<+\infty$, the following inequality holds:

$$
u\left(x_{1}, t_{1}\right) \leq u\left(x_{2}, t_{2}\right)\left(\frac{t_{2}}{t_{1}}\right)^{\frac{n a}{2}} \exp \left(\frac{a^{2} d^{2}\left(x_{1}, x_{2}\right)}{4\left(t_{2}-t_{1}\right)}+\frac{n a k}{2(a-1)}\left(t_{2}-t_{1}\right)\right)
$$

Proposition 4.2. (Global bounds) The sharp bounds on the heat kernel away from the walls of the Weyl chamber in Proposition 3.1 imply the same sharp bounds on the heat kernel in the region $|H| \leq \kappa(1+t)$; i.e. Theorem 2.1 follows from Proposition 3.1.

Proof. For every positive constant $\omega$, when $t \leq \omega$ and $|H| \leq \kappa(1+t) \leq \kappa(1+\omega)$, the sharp bounds on $h_{t}(\exp H)$ are well-known and follow from the general theory for the heat kernel of Riemannian manifolds (see $[\mathrm{K}]$ ).

On the other hand, there exists a constant $C$ such that, for any $H \in \overline{\mathfrak{a}^{+}}$, we can find $H^{\prime} \in \mathfrak{a}^{+}$satisfying $\inf _{\alpha \in \Sigma^{+}}\left\langle\alpha, H^{\prime}\right\rangle \geq \omega$ and $\left|H-H^{\prime}\right| \leq C$. Let $t^{\prime}=t+1$. Notice that when $|H| \leq \kappa(1+t)$,

$$
\left|\frac{|H|^{2}}{t}-\frac{\left|H^{\prime}\right|^{2}}{t^{\prime}}\right|=\mathrm{O}(1)
$$

By the first paragraph, we can assume that $t \geq \omega+1$. Then

$$
(1+t)^{\frac{m}{2}-\left|\Sigma^{++}\right|} \asymp \prod_{\alpha \in \Sigma^{++}}(t+\langle\alpha, H\rangle)^{\frac{m_{\alpha}+m_{2 \alpha}}{2}-1},
$$

and the sharp upper bound on $h_{t}(\exp H)$ follows from the bound on $h_{t^{\prime}}\left(\exp H^{\prime}\right)$ given in Proposition 3.1 by applying Proposition 4.1 to $u(x, t)=h_{t}(x), x_{1}=\exp H, x_{2}=\exp H^{\prime}$, $t_{1}=t, t_{2}=t^{\prime}$. The lower sharp bound on $h_{t}(\exp H)$ follows similarly by taking $t^{\prime}=t-1$.

Remark 4.3. As mentioned in Corollary 2.2, sharp bounds on the Green function [AJ1, Theorem 4.2.2] are derived from the sharp bounds on the heat kernel. Therefore, Proposition 4.2 gives an approach of obtaining sharp bounds on the Green function without using Trombi and Varadarajan's asymptotic expansion of spherical functions. Using the Harnack inequality for positive solutions of $\Delta u+\lambda u=0, \lambda \leq|\rho|^{2}$, we can also obtain sharp bounds on the Green function along the walls from the bounds away from the walls as above. 


\section{REFERENCES}

[A1] J.-Ph. Anker, La forme exacte de l'estimation fondamentale de Harish-Chandra, C. R. Acad. Sci. Paris Série I, 305 (1987), 371-374.

[A2] L L noyau de la chaleur sur les espaces symétriques $\mathrm{U}(p, q) / \mathrm{U}(p) \times \mathrm{U}(q)$, in Harmonic analysis, Luxembourg 1987, P. Eymard \& J.-P. Pier (eds.), Lect. Notes Math. 1359, SpringerVerlag (1988), 60-82.

[A3] Sharp estimates for some functions of the Laplacian on noncompact symmetric spaces, Duke Math. J. 65 (1992), 257-297.

[AJ1] J.-Ph. Anker \& L. Ji, Heat kernel and Green function estimates on noncompact symmetric spaces, Geom. Funct. Anal. (GAFA) 9 (1999), 1035-1091.

[AJ2] Comportement exact du noyau de la chaleur et de la fonction de Green sur les espaces symétriques non compacts, C. R. Acad. Sci. Paris Série I, 326 (1998), 153-156.

[BGV] N. Berline, E. Getzler \& M. Vergne, Heat kernel and Dirac operators, Springer-Verlag (1992).

[C] I. Chavel, Eigenvalues in Riemannian geometry, Academic Press (1984).

[D] E. B. Davies, Heat kernels and spectral theory, Cambridge Univ. Press (1989).

[G] A. Grigor'yan, Heat kernel of a noncompact Riemannian manifold, in Stochastic analysis, (Summer Research Institute, Cornell University, July 1993), M. C. Cranston \& M. A. Pinsky (eds.), Proc. Symp. Pure Math. 57, Amer. Math. Soc. (1995), 239-263.

[GJT] Y. Guivarc'h, L. Ji \& J. C. Taylor, Compactifications of symmetric spaces, Progr. Math. 156, Birkhäuser (1998).

[K] Y. Kannai, Off diagonal short time asymptotics for fundamental solutions of diffusion equations, Comm. P. D. E. 2 (1977), 781-830.

[LY] P. Li \& S. T. Yau, On the parabolic kernel of the Schrödinger operator, Acta Math. 156 (1986), 153-201.

[L] J. Lott, Heat kernels on covering spaces and topological invariants, J. Diff. Geom 35 (1992), 471-510.

[SY] R. Schoen \& S. T. Yau, Lectures on differential geometry, International Press (1994).

[T] J. C. Taylor, Martin compactifications, to appear in Topics in probability and Lie groups, J. C. Taylor (ed.), CRM Proc. Lect. Notes, Amer. Math. Soc..

[TV] P. C. Trombi \& V. S. Varadarajan, Spherical transforms on semisimple Lie groups, Ann. of Math. 94 (1971), 246-303.

[Va] N. Th. Varopoulos, The local theorem for symmetric diffusion on Lie groups - An overview, in Harmonic analysis and number theory, S. W. Drury and M. Ram Murty (eds.), Canad. Math. Soc. Conf. Proc. 21, Amer. Math. Soc. (1997), 143-152.

[Vl] V. S. Vladimirov, Methods of the theory of functions of many variables, M. I. T. Press (1966).

Jean-Philippe Anker, Université Henri Poincaré (Nancy I), Institut de Mathématiques Elie Cartan (Laboratoire Commun UHP-CNRS-INRIA), B.P. 239, F-54506 VandoeuvreLÈS-NANCY Cedex, France

E-mail address: anker@iecn.u--nancy.fr

Lizhen Ji, University of Michigan, Department of Mathematics, East Hall, 525 East University Avenue, Ann Arbor, MI 48109-1109, USA

E-mail address: 1ji@math.1sa.umich.edu 